Cahiers de philosophie de l'université de

\title{
Heidegger et l'Europe
}

Robert Uriac

\section{(2) OpenEdition}

Journals

Édition électronique

URL : https://journals.openedition.org/cpuc/1142

DOI : $10.4000 /$ cpuc. 1142

ISSN : 2677-6529

\section{Éditeur}

Presses universitaires de Caen

\section{Édition imprimée}

Date de publication : 15 décembre 2010

Pagination : 75-112

ISBN : 978-2-84133-365-3

ISSN : 1282-6545

Référence électronique

Robert Uriac, " Heidegger et l'Europe », Cahiers de philosophie de l'université de Caen [En ligne], 47 | 2010, mis en ligne le 02 septembre 2020, consulté le 31 janvier 2023. URL : http:// journals.openedition.org/cpuc/1142; DOI : https://doi.org/10.4000/cpuc.1142

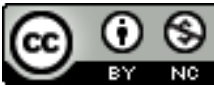

Creative Commons - Attribution - Pas d'Utilisation Commerciale 4.0 International - CC BY-NC 4.0 https://creativecommons.org/licenses/by-nc/4.0/ 


\section{Heidegger et l'Europe}

Ist das Sein ein bloßes Wort und seine Bedeutung ein Dunst, oder birgt das mit dem Wort «Sein» Genannte das geistige Schicksal des Abendlandes?

L'être est-il un simple vocable et sa signification une vapeur, ou bien faut-il dire que ce qui est désigné par le mot «être» abrite le destin spirituel de l'Occident $^{1}$ ?

Le vrai n'est pas pour tout le monde mais seulement pour les forts ${ }^{2}$.

Pour qui s'interroge sur l'élaboration de Sein und Zeit, le sentiment prévaut que la question de l'Europe ne joue guère de rôle dans la pensée de son auteur: le terme apparait peu, l'interrogation porte sur l'Être, la structure du Dasein dans son rapport à la temporalité en général. Il s'agit d'élaborer une ontologie fondamentale. Mais cette ontologie débouche aussi bien sur une théorie de l'historicité, et la lecture des textes postérieurs, notamment l'Introduction à la métaphysique, nous invite à remettre en question ce premier sentiment. Cette Introduction s'organise autour d'un enjeu clairement défini comme européen. Dans beaucoup d'autres textes, Heidegger pense, nous semble-t-il, le sens de l'activité philosophique dans la perspective d'une histoire qui est celle de l'Europe, lieu de la confrontation avec l'Être. Nous sommes par là invités à faire un retour en arrière et à découvrir que le chemin de cette pensée est sans doute en grande partie tracé par ce qu'on appelle à cette époque la "crise de l'Occident», et à mesurer la profondeur de l'engagement historique du philosophe.

La première moitié du $\mathrm{XX}^{\mathrm{e}}$ siècle, à travers deux guerres qui déchirent les peuples européens puis le monde entier, est en effet marquée par un ébranlement sans précédent de la civilisation occidentale. Par deux fois ces guerres décident du destin de l'Allemagne qui, au cœur de l'Europe, reste

1. M. Heidegger, Introduction à la métaphysique, Paris, Gallimard, 1967, p. 53; Einführung in die Metaphysik, Tübingen, Niemeyer, 1953 [cours de 1935], p. 32.

2. Ibid., p. 141; p. 102. 
vaincue après de redoutables initiatives. Heidegger, lui-même, se réfère au Déclin de l'Occident de Spengler, ou encore à Valéry, et sa pensée prend de plus en plus la forme d'une méditation sur le destin d'un Occident devenu planétaire mais dont l'Europe et surtout l'Allemagne et la Grèce, demeurent le centre, à la fois lumineux et énigmatique. Il y aurait donc un lien essentiel entre l'Europe et la Pensée, autre nom de la philosophie lorsque cette dernière échappe à la crise dont elle peut être la victime ou aussi le témoin, guettant la possibilité d'un "autre commencement » - l'Occident et l'Europe étant sans doute les deux noms d'une même question, mais avec un jeu qui, l'accentuant autrement, en déplace subtilement la signification.

\section{L'Europe, la philosophie, l'Université}

Dans le premier volume de Variété, Paul Valéry, précédant Husserl, considère la situation problématique de l'Europe, après la Grande Guerre. La civilisation européenne, expose-t-il, spirituelle, brillante, jusqu'alors en expansion, semble atteindre ses limites; elle est en crise et cette crise est fondamentalement "crise de l'Esprit»: «l'Esprit est en vérité cruellement atteint [...]. Il doute profondément de soi-même » ${ }^{3}$. L'Esprit qui, selon Goethe, «stets verneint », qui «nie toujours », qui est "avidité active, curiosité ardente et désintéressée». L'Europe a inventé la géométrie et, par là, introduit la pensée dans cette inquiétude inépuisable de la recherche du vrai qui bouleverse l'existence humaine. Husserl dira quelque chose d'équivalent, réfléchissant à la naissance de la géométrie puis de la philosophie, science universelle - «Urphänomen qui caractérise l'Europe au point de vue spirituel ${ }^{4}$ - fondatrice d'un nouveau type d'humanité et d'un processus de recherche indéfini.

Valéry nous propose en outre une généalogie qui met en évidence la complexité de cet esprit européen dont la destinée est en même temps planétaire. Un peuple est européen s'il a subi une triple influence: celle de Rome d'abord, du christianisme ensuite, lequel, issu du judaïsme, a rendu la conscience universelle, de la Grèce enfin, avec son modèle incorruptible de toute connaissance, "la géométrie »5. Mais cette complexité qui fait la richesse de l'esprit européen n'est-elle pas aussi ce qui amène l'Europe à s'opposer à elle-même et peut-être à se perdre? L'héritage constituant en effet une synthèse qui peut se défaire, chaque élément s'opposant aux

3. P. Valéry, Variété, Paris, Gallimard, 1924, p. 35.

4. E. Husserl, La crise de l'humanité européenne et la philosophie, Paris, Aubier, 1977, p. 3839.

5. P. Valéry, Variété, p. 53. 
autres dans une lutte pour la domination, cette lutte conduit aussi bien les peuples européens à la rivalité, à la guerre, et l'Europe en vient à se détruire elle-même. Nous sommes à ce moment décisif à partir duquel l'Europe peut disparaître ou au contraire se restaurer plus authentiquement. Telle est aussi l'interrogation de Heidegger en 1919, l'année où Valéry écrit $L a$ crise de l'esprit ${ }^{6}$.

Heidegger est en ce sens tout à fait européen. Catholique romain, il décide peu à peu de se consacrer entièrement à la science et à la science fondamentale, l'Urwissenschaft, la philosophie dont nous parle Husserl et dont l'origine est grecque. Les sciences et la philosophie se développent en Allemagne dans le cadre des universités nouvelles dont le prestige, au $\mathrm{XIX}^{\mathrm{e}}$ siècle, est considérable. Comme catholique, il s'inscrit dans l'héritage de la chrétienté et donc de l'Europe - "Christenheit oder Europa», écrivait Novalis en 1899 -, mais cet héritage est fort complexe: peut-être celui d'une parole dévoilante, recueillie dans la Bible, annonciatrice d'un salut, mais aussi celui d'une lourde tradition cherchant à se maintenir et s'opposant à la modernité, scientifique, technique, démocratique (autre visage de l'Europe). Heidegger est d'abord ce catholique traditionnel qui pense la modernité européenne comme risque d'une perte fondamentale; l'homme moderne dont l'existence est «horizontale» est menacé "par une insécurité croissante et une totale désorientation ${ }^{7}$. Le catholicisme dans lequel il grandit invite à faire retour vers la foi médiévale et son élaboration philosophique. À travers la scolastique, et notamment l'étude de Duns Scot, nous sommes renvoyés à l'examen philosophique de la question de la signification, puis à la question de l'Être en référence aux analyses d'Aristote, autre parole initiale. Le catholicisme à l'intérieur duquel Heidegger développe sa pensée est synthèse conflictuelle de Jérusalem, d'Athènes et de Rome.

Cette synthèse va peu à peu se défaire. Heidegger renonce à sa vocation première et s'éloigne du catholicisme; il rejoint l'université et son objectif principal sera de s'y faire reconnaître, d'accéder au poste de professeur pour se consacrer à l'enseignement et à la recherche philosophique. La rupture avec le catholicisme est violente - ce dernier lui apparaissant désormais comme un carcan dogmatique dont il faut se libérer - mais elle n'est peutêtre pas totale: il reste chrétien, en communion d'esprit avec sa femme, se convertit au protestantisme, approfondit sa lecture de la Bible et travaille les écrits du jeune Luther dont il dira qu'il est son guide à l'égal d'Aristote. «Celui qui m’a accompagné dans mes recherches était le jeune Luther et

6. Ibid., p. $11 s q$.

7. H. Ott, Martin Heidegger. Éléments pour une biographie, Paris, Payot, 1990, p. 118. 
mon modèle était Aristote", écrit-il ${ }^{8}$. Mais la rupture va se radicaliser: la philosophie est une libre recherche étrangère à tout présupposé fourni par la foi. Et le fil conducteur de Luther sera lui aussi abandonné.

Heidegger se tourne donc vers l'université. Le mobile religieux n'a cependant pas disparu et l'attente spirituelle de celui qui médite à partir du luthérien Kierkegaard n'est pas satisfaite. L'idéal philosophique et scientifique sur lequel reposent la recherche et l'enseignement universitaires aboutit à une fragmentation de la science qui se préoccupe uniquement d'elle-même et s'est séparée de la vie concrète et existante; elle est considérée comme une activité désintéressée, elle est le produit d'un intellect desséché et privé de sol. Nécessité alors de refuser l'éclatement des sciences en une pluralité éparpillée de spécialités dominées par un modèle naturaliste, auquel on ne pourrait échapper que par la théorie de la science néo-kantienne et son sujet transcendantal. Il faut retrouver l'unité, le fondement, un enracinement dans la vie, bref échapper à la Bodenlosigkeit. Dans ce but il faut réexaminer le sens des propositions et l'idée de la vérité, étudier la logique et restaurer l'ontologie, poser à nouveau la question originaire de l'Être, à partir de laquelle le sens de ses différentes régions se trouve éclairé. Cette question est oubliée. Heidegger cherche donc plus loin, en direction des "choses mêmes", mais aussi de ce qui est enfoui dans notre mémoire, l’oubli étant une modalité du souvenir. La phénoménologie de Husserl lui apparait comme une direction possible et semble lui donner la possibilité d'élucider le sens des propositions en faisant retour aux choses elles-mêmes. Permet-elle cependant la remémoration?

\section{L'Université et la guerre. La vie faktisch-historisch}

La question va prendre une tout autre acuité avec la guerre qui confronte les peuples européens à leur avenir. Heidegger participe aux opérations à l'arrière de Verdun et, réfléchissant, en mars 1916, au contraste entre son semestre universitaire passé et ce qu'il vit actuellement, il écrit à sa femme:

je sais aujourd'hui qu'une philosophie de la vie vivante a le droit d'exister, que j'ai le droit de déclarer au rationalisme une guerre à couteaux tirés sans subir l'anathème de la non-scientificité, j'en ai le droit et je le dois [...]. Nous ne devons pas donner à nos jeunes héros qui reviennent affamés du champ de bataille des pierres au lieu de pain, nous ne pouvons leur proposer des

8. M. Heidegger, Ontologie. Hermeneutik der Faktizität, Francfort, Klostermann (Gesamtausgabe [désormais GA]; 63), 1988, p. 5 (nous traduisons). 
catégories mortes, des formes qui ne sont que des ombres, des fonds de tiroir exsangues où conserver bien soigneusement et y laisser moisir une vie usée par le rationalisme ${ }^{9}$.

La guerre nous renvoie donc à nous-mêmes et au souci de notre être, elle nous invite à prendre soin de nous sans nous égarer dans un vain savoir, œuvre de la raison et d'une philosophie d'autrefois; nous sommes invités à nous découvrir non pas comme intellect ou raison mais comme esprit, agissant aujourd'hui. Ce radicalisme philosophique est en même temps projet révolutionnaire: c'est toute la science allemande qu'il faut transformer, en transformant l'existence allemande et réciproquement: «völlige Umwälzung unseres deutschen Daseins ${ }^{10}$.

Une autre lettre de cette période montre bien la portée de cette opposition entre l'intellect et l'esprit dans son rapport avec la question de l'université considérée comme le lieu où se développe le «rationalisme». En juin 1918, il est affecté à Berlin et se rend dans le cœur de la ville. Il écrit:

nous avons observé l'animation de la Friedrichstrasse, nous sommes repartis chez nous complètement écœurés... C'est une folie telle que je n'aurais pas pu imaginer cela; dans un tel milieu il ne peut y avoir de véritable culture de l'esprit [...]. La guerre n'a pas encore été pour nous assez terrible. Les hommes ici ont perdu leur âme - les visages n'ont plus aucune expression - tout au plus celle de la vulgarité. Cette décadence ne connaît plus aucun frein, peut-être pourra-t-on venir à bout du Berlin « intellectuel » grâce à une culture enracinée dans son terroir dans les universités de province - en tout la guérison de notre jeunesse ne viendra que de là -, à supposer qu'elle soit encore possible ${ }^{11}$.

L'expérience de la guerre nous reconduit par conséquent à la question des tâches de l'université, mais cette dernière est investie d'un rôle considérable, aller jusqu'au bout de la guerre, car «la guerre n’a pas été pour nous assez terrible», puis tirer de cette radicalisation la possibilité d'une guérison, d'une régénération de l'esprit considéré dans sa globalité. Il ne s'agit plus ainsi de transformer la seule université, mais d'en faire le lieu où, par la radicalité du questionnement, la vie de l'esprit peut se régénérer en Allemagne. L'université s'est aliénée; elle s'est laissée détourner d'ellemême, de sa vocation authentique, elle est emportée par la curiositas; elle ne délivre qu'un pseudo-savoir, des «catégories mortes, exsangues»; il faut

9. M. Heidegger, "Ma chère petite âme» : lettres à sa femme Elfride, 1915-1970, Paris, Seuil, 2007, p. 66. Lettre du 5 mars 1916.

10. M. Heidegger, Reden und andere Zeugnisse eines Lebensweges, Francfort, Klostermann (GA; 16), 200o, p. 184.

11. M. Heidegger, «Ma chère petite âme» : lettres..., p. 109. Lettre du 21 juillet 1918. 
affronter la mort, retourner la mort contre elle-même; l'université rendra alors possible la restauration souhaitable, ce que Heidegger appelle dans la lettre précédemment citée «la guérison», évoquant la parole évangélique "qui veut sauver sa vie la perdra». Le Spiessburger doit être vaincu, il faut combattre «à couteaux tirés» afin de révolutionner la vie universitaire allemande, la retourner complètement, en recherchant un point d'appui, un sol solide.

Il s'agit de bouleverser radicalement l'existence allemande. Un texte de 1934 peut sans doute éclairer rétrospectivement la dimension fondamentale de ce moment critique. Il s'agit d'un passage d'une conférence consacrée, précisément, à l'Université allemande ${ }^{12}$; Heidegger retrace l'histoire de la nouvelle université allemande, non pas l'université médiévale, mais l'université allemande autochtone, prussienne et völkisch. Or le moment décisif de cette histoire est la guerre et l'assomption de la défaite: cette dernière n'a rien décidé car la décision appartient à chacun et au peuple, à chacun dans et à travers le peuple. La guerre nous fournit l'expérience d'une nouvelle communauté qui n'est pas la société bourgeoise fondée sur la raison sans principe et les droits de l'individu égoïste, les prétendus droits de l'homme et la théorie du contrat social. À travers la guerre le peuple allemand est appelé à accomplir enfin sa vocation, il est convoqué à redevenir ce qu'il est vraiment, à exister authentiquement: il se découvre alors comme le peuple de l'esprit: «l'esprit du front est la volonté savante d'une communauté nouvelle [...]. L'esprit du front signifie justement la conquête spirituelle et la transformation créatrice de la guerre». La guerre est ainsi pensée comme régénération spirituelle, régénérant aussi bien le peuple que le savoir car le savoir est toujours pensé comme savoir de soi: transformer son savoir revient à se transformer soi-même.

Se donnant le but d'élaborer la science dans un horizon de rationalité, l'université fonctionne, en effet, sous le régime de l'objectivité, ce qui implique d'abord l'orientation du savoir en fonction d'un objet visé, puis la détermination de la vérité comme saisie de l'objet, adéquation à l'objet, enfin la pluralité des sciences, conséquence de la pluralité des objets. Mais dans un tel processus, l'être connaissant s'oublie lui-même, ou se pense comme sujet transcendantal à partir de la distinction sujet-objet, et le Soi s'est alors déjà perdu, détourné de lui-même dans la préoccupation affairée. Il est devenu incapable de se saisir et de se reconquérir. Or le savoir n'est pas uniquement savoir d'objet mais m'éclaire aussi sur moi dans mon existence concrète et constitue une certaine façon de m'oublier ou de prendre soin

12. M. Heidegger, Reden und andere... (GA; 16), p. 285 sq. 
de moi. La phénoménologie avec sa théorie de l'intuition semble offrir la possibilité de saisir l'existence concrète mais, elle aussi, est bien vite récusée: elle est certes retour aux choses mêmes, sa démarche, toutefois, reste marquée par la problématique théoricisante de l'ego.

Si la tâche de la philosophie est de retrouver la vie dans son mouvement effectif et de refonder la science à partir d'une science première qui s'enracine dans le mouvement originaire de la vie, la phénoménologie ne peut suffire.

Je ne peux accepter la phénoménologie, comme chose définitive, car elle est dans son point de départ et conséquemment dans son but, trop étriquée et trop exsangue et parce qu'une telle manière d'aborder les choses ne peut être absolutisée. La vie est trop riche et trop grande [...]. Cet hiver «l'homme historique» m'[est] apparu dans un éclair ${ }^{13}$.

«Que j'en sois venu à Husserl n'est qu'épisode». La phénoménologie reste extérieure à l'historicité, elle n'est pas à la hauteur de la crise, et l'être humain que je suis, que nous sommes, existe historiquement ${ }^{14}$. L'intérêt pour la pensée de Husserl ne peut être qu' «épisodique».

Mais les différentes pensées de l'histoire elles-mêmes, la Kulturkritik de Spengler, Simmel, Scheler, l'herméneutique diltheyenne, envisagent le passé et le processus historique de manière trop extérieure en construisant des types abstraits qui objectivent le devenir. De ce fait, bien que s'efforçant de penser l'histoire, ces auteurs méconnaissent la nature de l'histoire et du passé; ce dernier n'est pas ce qui a disparu, n'existe plus, et qu'il s'agit de reconstituer par une enquête objective, il est là, en nous et nous sommes en lui; nous sommes intérieurs à l'histoire qui nous est également intérieure. S'il faut trouver un sol, celui-ci nous est fourni par la vie, non pas celle des biologistes, ni celle des "philosophies de la vie», mais celle qui est en même temps esprit, la vie historique concrète de ces hommes que nous sommes, toujours déjà là avant même que nous ayons commencé à réfléchir. C'est de là que provient notre pensée, c'est là qu'elle trouve sa source et le principe de son mouvement, puisque la vie cherche à s'élucider, visant son propre sens. Il faut nous installer en elle, refusant tout au-delà, rester, en un sens nietzschéen, «fidèles à la terre». Heidegger va appeler cette vie, la "vie factice» ou "facticité»,

13. M. Heidegger, «Ma chère petite âme» : lettres..., p. 89-90. Lettre du dimanche de l'Ascension 1917.

14. Je traduis « Geschichtlichkeit, geschichtlich» par «historicité, historique», suivant l'argumentation de J. A. Barash, Heidegger et son siècle, Paris, PUF, 1995, p. 17-18. Voir également Heidegger et le sens de l'histoire, Paris, Galaade Éditions, 2006. 
c'est-à-dire, à la fois, la vie telle qu'elle est en fait et telle qu'elle se fait en se comprenant elle-même. La vie surmonte l'opposition du sujet et de l'objet, elle est mouvement, dépassement de soi. Elle est toujours particularité, car n'existent que des vivants. Elle est relation, car un vivant n'est jamais isolé en sa pure existence. Je suis Dasein, là, vivant non comme un animal mais existant dans l'ouverture d'un horizon de sens à partir duquel un monde spirituel, historique, se constitue et configure ma vie. Il s'agit pour Heidegger d'entreprendre une «herméneutique de la vie facticielle ", c'est-à-dire de s'installer au cour de cette vie en essayant de découvrir comment celle-ci se donne à elle-même sa propre lumière. La bonne attitude est de ne pas sortir de la vie elle-même car il n'y a pas de sujet qui surplombe la vie: la philosophie est auto-interprétation de la vie, tel est le cercle herméneutique fondamental.

Ainsi se trouve résolu ce que Heidegger nomme le «problème Münchhausen " : sortir ${ }^{15}$ des sables mouvants en prenant appui sur soi-même. Descartes est une sorte de baron de Münchhausen dans la mesure où il part de son incertitude et s'efforce de trouver la solution en soi-même, comme si le doute produisait sa lumière et nous donnait un point d'appui inébranlable qui se soutiendrait lui-même. Mais il ne soutient précisément que lui-même et ne tient pas sur le sol. C'est ce que Heidegger appelle une subjectivité exsangue, fermée sur elle-même. La solution consiste au contraire à se penser d'emblée dans la relation dans laquelle je suis toujours déjà jeté: l'homme historique est toujours déjà là dans un monde qu'il n'a pas constitué et à partir duquel se définissent des possibilités d'existence. Être européen notamment, c'est être pris dans une histoire, être jeté [geworfen], dans un déjà-là, et aussi bien projeté vers un avenir; je suis par exemple confronté à la guerre et incité à me poser la question de l'avenir de l'Allemagne. La vie existe toujours déjà avant moi. Si je cherche à me penser, moi-même et ma vie, en la reconstruisant comme si je lui étais extérieur, je me saisis nécessairement au moyen d'abstractions, de pensées solidifiées, considérant les choses dans l'horizon de la présence permanente et recherchant une vérité hors du temps. Ne serait-ce pas, comme le disait Nietzsche, parce que j'aurais perdu ma propre vitalité?

Ma propre interrogation provient de la vie, mais la vie contient aussi en elle la possibilité de se méconnaître, de devenir étrangère à elle-même. Ce schéma fondamental est, semble-t-il, au principe de la démarche de

15. M. Heidegger, Concepts fondamentaux de la métaphysique, Paris, Gallimard, 1992, p. 43; Die Grundbegriffe der Metaphysik, Welt - Endlichkeit - Einsamkeit, Francfort, Klostermann (GA; 29-30), 1983. 
Heidegger et semble étonnamment proche du thème augustinien de la conversion, au livre 10 des Confessions ${ }^{16}$, où Augustin montre comment l'esprit se perd dans l'extériorité, attiré par la multiplicité des créatures. Il s'oublie lui-même et en même temps Dieu qui est en lui, au principe de sa vie et de sa recherche du savoir. Mais l'oubli est une modalité de la mémoire. L'Être n'est donc jamais perdu dans la mesure où toute recherche est accomplie à partir d'un mouvement venant de l'Être divin lui-même et le visant implicitement. De la même façon, le Dasein s'oublie dans la science comme étude désintéressée, alors qu'il se cherche lui-même, non pas certes dans sa pure égoïté, mais comme celui qui a souci de l'Être et de la vérité, ouvrant par-là un monde. S'oublier est indissociablement oubli de soi comme être-au-monde et oubli de l'Être. Cet Être oublié est cependant déjà là dans le moindre des mouvements du Dasein, de cet existant factice-historique que nous sommes, ce pourquoi notre vie n'est pas animale mais spirituelle, traversée par le souci. Il appartient certes à cette existence de pouvoir se perdre, mais dans cette perte même, la source dont elle provient se trouve encore indiquée. Il n'est alors pas étonnant que l'université trahisse sa mission, la chute [Verfall], étant constitutive du mouvement de l'existence: l'université se meut dans la pensée toute faite, le On, la sécurité des carrières et du savoir objectif, bref l'inauthenticité. Il faut oser rompre avec cette inauthenticité, "se battre à couteaux tirés", affronter l'angoisse et l'incertitude, puis refaire le chemin, en sens inverse, en retournant la pensée la plus abstraite afin de découvrir à partir d'elle, remontant la pente, l'originaire masqué, mais encore présent dans son absence même.

\section{La déconstruction: Destruktion et Abbau}

Reprendre l'héritage de cette vieille Europe et le détruire ou le déconstruire: dans une lettre de 1920 à son élève Löwith ${ }^{17}$, Heidegger explique que son but n'est pas de se livrer à l'activité générale de la culture pour «sauver la culture», mais "par un démontage et un remontage radicaux, par une destruction acquérir la solide conviction de ce "Un qui est nécessaire" 18 sans se soucier des commentaires des gens doués et zélés qui mesurent le temps avec une horloge»; il faut prendre «le risque d'être broyé [...] dans la situation révolutionnaire que je vis aujourd'hui sans me préoccuper s'il en sortira une culture ou une accélération du déclin». La Destruktion est

\footnotetext{
16. Saint Augustin, Confessions, livres 9-13, Paris, Les Belles lettres, 1969, p. 250 sq.

17. K. Löwith, Ma vie en Allemagne avant et après 1933, Paris, Hachette, 1988, p. 45.

18. On peut remarquer une référence évangélique implicite.
} 
donc une opération risquée qui peut prendre un tour purement nihiliste en mettant en évidence l'inanité de tout ce qu'on appelle la culture, mais son secret espoir est la découverte d'un sol enfin véritable.

Considérée de ce dernier point de vue, elle devient déconstruction [Abbau], et consiste à examiner la tradition non pas pour ce qu'elle se donne mais en essayant de découvrir, à partir d'elle, quelque chose de plus profond qu'elle révèle en le dissimulant. Heidegger va faire subir ce traitement à son héritage chrétien. Il examine le jeune Luther, non pas pour étudier la théologie protestante, mais l'expérience chrétienne de la vie considérée comme expérience de la vie factice. Il cherche à découvrir la vie dans son noyau originaire avant qu'elle ne soit recouverte par une théorie qui en fausserait le sens, démarche prolongée par une étude des Épîtres aux Thessaloniciens de saint Paul et du livre 10 des Confessions de saint Augustin ${ }^{19}$. Heidegger se propose de déconstruire le discours théologique chrétien en isolant un noyau chrétien primitif altéré par l'hellénisme: la Grèce comme masque de la saisie chrétienne de la vie, tel est le point ici remarquable.

Heidegger ne se propose toutefois pas de transposer philosophiquement le christianisme, mais essaye de montrer qu'il est possible de trouver dans les textes de Paul ou d'Augustin une expérience de la vie factice plus authentique que celle que l'on trouve chez les Grecs dont le sens est faussé par les concepts, platoniciens ou aristotéliciens, de substance - présence permanente. La recherche vise une saisie de la temporalité et de la temporalisation qui est celle de l'existence comme telle. On peut la découvrir à travers la description de la vie chrétienne, tendue vers la promesse de la venue du Messie ou encore dans les textes augustiniens concernant le souci. Certes, le futur de la promesse doit être pensé ontologiquement, mais il n'en reste pas moins que le sens authentique du futur se trouve élaboré à partir de l'annonce de la venue du Messie à la fin des temps! On peut alors se demander si ce schéma kérygmatique à partir duquel Heidegger élabore sa conception du temps et de l'historicité ne subsiste pas durablement et n'oriente pas, implicitement, sa conception du rapport du peuple allemand au peuple grec - en particulier pour ce qui est de sa perspective d'un bouleversement radical de l'existence de tout un peuple, «die völlige Unwälzung unseres deutschen Daseins ${ }^{20}$. Par-delà le refus explicite du christianisme, la pensée heideggerienne de la «révolution» serait ainsi marquée par le messianisme chrétien.

19. M. Heidegger, Phänomenologie des religiösen Lebens, Francfort, Klostermann (GA; 60), 1995.

20. M. Heidegger, Reden und andere... (GA; 16), p. 184. 
Comme dans la théologie dialectique de Barth, Gogarten et Bultman, cette démarche prolonge la critique de Kierkegaard. Barth considère que le protestantisme allemand s'est sécularisé et a de ce fait perdu le sens radical de la transcendance divine en raison d'une chute dans la quotidienneté. Il s'agit pour lui de retrouver le noyau kérygmatique, ce qui suppose la mise en perspective de la vie humaine avec une temporalité primordiale, un avènement annoncé qui n'est pas de l'ordre de ce qui arrive au sein du monde. Le message suppose une disponibilité absolue et proprement révolutionnaire, il dissout le monde dans sa totalité pour nous référer à une altérité radicale à partir de laquelle l'existence prend son sens. Une telle perspective est partagée par Heidegger, mais ce dernier refuse de soumettre la philosophie à la théologie, sa visée étant au contraire d'écrire une ontologie: ce qui transcende tout étant n'est pas Dieu, étant éminent parmi d'autres, mais l'Être comme condition de possibilité de la saisie de l'étant. L'Être est "transcendant», mais non pas éternel, dans un au-delà du monde. Il est historique, historicité et histoire, d'ordre « existential». La pensée d'une temporalité originaire, distincte du temps ordinaire, et l'idée que cette temporalité n'est pas quelque chose d'extérieur à nous-mêmes reste commune aux deux perspectives. Elle conduit à considérer qu'il ne s'agit nullement de faire une théorie de l'Être et du temps, mais de provoquer un bouleversement radical dans notre manière d'être en découvrant ce qui se joue à travers la temporalité.

La mise en évidence de la temporalité authentique du Dasein se prolonge dans la confrontation avec Aristote et une déconstruction de l'ontologie aristotélicienne, les deux projets étant étroitement reliés comme le montrent par exemple le rapport Natorp ${ }^{21}$ et les textes des cours consacrés à une interprétation phénoménologique d'Aristote. Heidegger relit la théorie aristotélicienne de la substance, du mouvement et du temps, pour découvrir comment la conception de l'Être comme présence subsistante provient de cette temporalité originaire mais en la méconnaissant. La démarche aboutit au livre fondamental, Sein und Zeit, publié en $1927^{22}$. Ce dernier s'efforce de dégager cette temporalité originaire à partir de l'analyse du Dasein, de montrer comment la conception ordinaire du temps en provient par un processus de chute, de dégradation. Il en résulte une nouvelle conception de l'être humain et de la temporalité en général, qui préfigure une ontologie, théorie générale de l'être en tant que tel. On peut alors avoir le sentiment que Heidegger s'éloigne complètement de l'Europe, qu'il s'occupe d'une

21. M. Heidegger, Interprétations phénoménologiques d'Aristote, J.-F. Courtine (trad.), Mauvezin, Trans-Europ-Repress, 1992.

22. M. Heidegger, Sein und Zeit, Tübingen, Niemeyer, 1972. 
construction conceptuelle extrêmement complexe, dont l'objet est de penser à nouveaux frais ce qu'on peut appeler la réalité humaine et, à travers l'existence de l'homme, d'élucider le sens de l'Être et de la temporalité en général, de telle sorte que tout individu, quel qu'il soit, est invité à se confronter à la mort et à se découvrir dans son authenticité. Mais une telle impression doit être dissipée. Le but de Heidegger n'est pas de nous parler de la réalité humaine. Il n'y a pas d'humanité en général si on entend par «humanité» la totalité des êtres humains ou encore l'ensemble des caractères communs à tous les hommes et les distinguant des autres êtres. Ce que nous nommons «homme» est le Dasein or celui-ci existe toujours dans un lieu et un temps singulier. Au reste, l'Être [das Sein], est lui aussi singulier et le saisir comme un universel revient à le ramener à un simple mot qui le fait disparaître dans l'oubli. L'Être est donc histoire et l'histoire est histoire de l'Être. Si Rome invente en même temps l'Histoire universelle avec Polybe et l'humanitas, l'humanité, selon la Lettre sur l'humanisme ${ }^{23}$, cette invention est méconnaissance et oubli. Il n'y a pas de Weltgeschischte mais une Seinsgeschichte.

\section{Historicité et répétition}

La théorie de l'historicité couronne à juste titre le maitre-livre ${ }^{24}$. L'aboutissement de la déconstruction est le retour à l'histoire, retour à l'existence dans un peuple, et la reprise d'une tradition qui se choisit ses héros. La notion de répétition nous apparaît ici décisive. Selon Heidegger, en effet, mon existence présente reçoit son sens d'un passé singulier dont elle provient, le monde au sein duquel elle se construit ayant déjà été configuré. Or en tant que je suis dans un monde, toujours déjà, en lui, jeté, je me rapporte aux éléments de ce monde, oubliant sa configuration, c'est-à-dire l'opération par laquelle seulement, il est devenu ce monde. Il est donc nécessaire de répéter cette institution originaire, de reprendre activement la mondanéisation du monde. Le sens du futur en dépend, puisque la configuration du monde ouvre sur des possibles qui ne le sont devenus que par elle. La démarche aboutit alors à une double conséquence: la position d'un originaire, d'une fondation, et l'accent mis sur la problématique de la répétition.

Répéter peut ici vouloir dire deux choses: reproduire en moins bien, en altérant, en oubliant le sens, alors même qu'on croit le préserver; ou, tout au contraire, restaurer la signification originaire, et par conséquent, ouvrir à nouveau les possibles et faire jaillir le futur dans la plénitude de

23. M. Heidegger, Lettre sur l'humanisme, éd. bilingue, Paris, Aubier, 1964, p. 47.

24. M. Heidegger, Sein und Zeit, p. 382 sq. 
cette ouverture, à partir d'un passé restitué à lui-même. Cette opposition ne constitue-t-elle pas pour Heidegger l'alternative fondamentale? Si le passé persiste dans le présent aveuglément, restaurons-le dans la plénitude de sa signification! L'Allemagne est notre tradition, notre être le plus intime, elle s'est perdue, elle est devenue étrangère à elle-même. Elle a perdu son sol originaire ou, plutôt, elle l'habite mais en lui étant infidèle; elle se doit donc de retrouver ce dont elle provient dans la singularité de son être. Par là, son futur s'ouvrira à nouveau, elle sera à nouveau elle-même dans la plénitude de son être, comme elle y est appelée par son destin. L'histoire n'est pas nécessité mécanique, enchaînement des générations, réalisation progressive de la Liberté ou de l'Esprit, elle est destin. Nul n'échappe à son destin, mais il est possible de lui être fidèle ou infidèle, de l'assumer ou de le trahir. Le philosophe, lui, va devenir prophète ou devin: il est celui qui rappelle le peuple à sa vocation, à son destin, celui qui restaure l'origine et exige fidélité du peuple à lui-même, en évoquant «l'autre commencement». Heidegger est ainsi comme un Zarathoustra du peuple allemand: non pas celui qui demande fidélité à la terre mais à l'Allemagne.

Fidélité de l'Allemagne à elle-même, tel est sans doute le point de départ de l'aventure du Rectorat. Au sujet de Hölderlin, Heidegger écrira:

Les poètes [...] sont prophétiques. Mais ce ne sont pas des prophètes au sens judéo-chrétien du mot. Les prophètes de ces religions [...] annoncent aussitôt le Dieu sur lequel on comptera ensuite comme garantie du salut dans la béatitude supraterrestre. Qu'on ne défigure pas la poésie de Hölderlin avec le religieux de la religion qui demeure l'affaire de la façon romaine d'interpréter les rapports entre les hommes et les dieux [...]. Le sacré qui est dit dans la prédiction poétique ne fait qu'ouvrir le temps d'une apparition des dieux, et qu'indiquer la région où se situe la demeure sur cette terre de l'homme requis par le destin de l'histoire ${ }^{25}$.

Mais quel passé? Comment déterminer ce destin? Le passé est vaste, comment découvrir l'originaire? «Il faut choisir ses héros», dit Heidegger. Le héros est le combattant victorieux, celui que la gloire acquise au combat inscrit à jamais dans la mémoire des hommes, mais il est aussi ce demi-dieu fondateur dont on raconte comment il a institué les choses qui sont aujourd'hui, le configurateur d'un monde, celui dont la «frappe» [Prägung], a institué à l'origine les choses comme elles sont, en tant qu'elles constituent un monde. Or la geste du héros est racontée dans le mythe. Et le mythe ne doit pas être oublié, non seulement il doit être récité, mais encore perpétuellement réactivé, afin que son sens ne soit pas perdu. L'histoire

25. M. Heidegger, Approche de Hölderlin, Paris, Gallimard, 1973, p. 145. 
selon Heidegger n'est pas l'historiographie objective mais une mythologie, dans la perspective tracée par Nietzsche (deuxième Intempestive ${ }^{26}$ - unique référence à Nietzsche de Sein und Zeit $\left.{ }^{27}\right)$. La Geschichte ne conduit pas à une Historie mais à un Mythe. Étrange retournement de perspective: la recherche de la vie effective-factice-historique aboutit à la construction d'une mythologie ou plutôt à la recherche dans le passé d'une dimension mythique. La démarche n'est plus ici augustinienne, elle remonte par-delà le christianisme à un primitivisme radical comparable aux recherches des poètes et des peintres expressionnistes de cette époque qui veulent régénérer la vieille civilisation européenne:

Le commencement est ce qu'il y a de plus inquiétant et de plus violent [...]. Le caractère inexplicable de ce commencement ne tient pas à une insuffisance ou à une impuissance de notre intelligence de l'histoire. Au contraire, c'est dans la compréhension du caractère mystérieux de ce commencement que résident l'authenticité et la grandeur de la connaissance historique. La connaissance de l'histoire à ses origines ne consiste pas à déterrer le primitif et à rassembler ses ossements. Elle n'est pas une science de la nature totalement, ni même à moitié; si elle est quelque chose, c'est une mythologie ${ }^{28}$.

Toutefois, la question rebondit: quels sont ces héros? Non pas les Naturvölker, non pas les Germains, mais les Grecs. La réponse de Heidegger est ici originale et le distingue incontestablement des expressionnistes et des idéologues du parti national-socialiste: ces héros fondateurs sont plus particulièrement les philosophes présocratiques. Sein und Zeit ne peut pas être achevé parce que l'historicité nous fait sortir de l'entreprise de déconstruction d'Aristote et de Platon pour remonter jusqu'à un originaire qui n'est plus la facticité de la vie, mais la Grèce. Il s'agit maintenant de traduire et de répéter cet originaire qui constitue la fondation de l'Occident comme Esprit. La garde de cet Esprit nous a été confiée. L'Esprit peut se définir comme l'ouverture de l'Être. L'Être est donc purement occidental. Il s'ouvre en ouvrant l'Occident comme Histoire et Destin. Or l'Allemagne, dans la mesure où elle occupe le centre de l'Occident, a reçu cette garde. Il faut donc comprendre que l'Allemagne, à l'intérieur de l'histoire de l'Être, a reçu du destin la mission de restaurer l'origine grecque de cette histoire, constitutive de l'Occident.

26. F. Nietzsche, Considérations intempestives II, éd. bilingue, Paris, Aubier, 1964, p. 303.

27. M. Heidegger, Sein und Zeit, p. 434.

28. M. Heidegger, Introduction à la métaphysique, p. 162. Heidegger entend ici le primitif au sens où l'ethnologie en fait un stade élémentaire de l'humanité dépassé par le progrès de la civilisation. Sa position est néanmoins primitiviste, au sens où elle vise un retour à un état primitif, lequel apparaît comme fondation originaire: ce qui est primitif n'est pas «arriéré, maladroit, et faible. En vérité c'est le contraire qui se produit» (ibid., p. 119). 


\section{Les Grecs, l'origine grecque et la grande décision}

Dans les années trente, Heidegger reprend la lecture des Présocratiques, sa référence aux Grecs va changer: remplaçant Aristote, les Présocratiques vont occuper le premier plan. Il ne s'agira plus seulement de déconstruire mais de rétablir une fidélité à l'origine. La déconstruction porte sur ce qui masque ou éloigne, par exemple la philologie. Ce déplacement décisif permet de mettre en perspective l'analytique du Dasein avec l'engagement de la période du Rectorat, précédé par ce changement de style philosophique. La production philosophique de Heidegger dans cette période prend la forme de conférences, où l'herméneutique du Dasein, la déconstruction d'Aristote $^{29}$, laissent la place à l'autre Grèce, celle des Présocratiques et des grands tragiques, de Sophocle tout particulièrement. Cette démarche se développe au moyen de traductions et de commentaires des paroles fondamentales des Grecs - Platon étant soupçonné, dans une perspective que l'on trouve déjà chez Nietzsche, d'avoir rompu avec la Grèce originaire.

La correspondance avec Elisabeth Blochmann, durant ces années décisives, montre le déplacement: Heidegger écrit à Elisabeth qu'il a abandonné Être et temps II, ce texte n'étant qu'un «chemin et ce chemin n'est plus foulé depuis longtemps et il est même recouvert »: «Je ne peux plus du tout écrire Etre et temps II, je n'écris d'ailleurs aucun livre ${ }^{30}$, précise-t-il d'ailleurs. Cette lettre prend la suite d'une autre dans laquelle il commente son cours sur L'essence de la vérité et s'efforce d'en dégager l'orientation essentielle:

Il m’apparait que le commencement de notre philosophie occidentale doit redevenir en nous présent afin qu'en présence d'un tel modèle nous puissions commencer véritablement à apprendre que tout un chacun n'a nullement les titres requis pour accomplir n'importe quoi.

Il ajoute: «Déployer notre être à la mesure d'une histoire ne peut surgir que d'une parole venue de l'Antiquité». Il récuse les livres et l'écriture et, dans le même mouvement, postule l'existence d'un rang ou d'une distinction qui habilitent certains à «accomplir » ce qui doit être accompli : la refondation de notre pensée.

La lettre fondamentale du 30 mars 1933 précise, en effet, l'objectif poursuivi et indique le rapport que Heidegger entrevoit entre les événements de 1933, ce qu'il appelle le mouvement, et le commencement de la philosophie

29. M. Heidegger, Phänomenologische Interpretationen zu Aristoteles, Francfort, Klostermann (GA; 61), 1985.

30. M. Heidegger, Correspondance avec Elisabeth Blochmann, Paris, Gallimard, 1996, p. 270. Lettre du 18 août 1932. 
grecque: «il y a longtemps que le caractère délavé et fantomatique d'une culture et l'irréalité de prétendues valeurs sont devenus à mes yeux nuls et non avenus, d'où ma recherche d'un nouveau sol dans le Dasein. Nous ne trouverons ce sol, et avec lui la vocation des Allemands, que si nous nous exposons à l'Être lui-même d'une façon et par une appropriation neuves. Ainsi, c'est entièrement à partir de l'avenir que nous éprouvons le présent», mais cet avenir est lui-même mesuré par un passé fondateur qu'il a pour tâche de répéter; ne faire aucune place aux Grecs dans la situation actuelle, comme le fait par exemple Jaspers, «équivaut à une catastrophe aujourd'hui en cette heure du monde de l'aventure occidentale $»^{31}$. Il s'agit là «du destin mondial de notre peuple».

On pourrait faire remarquer que voulant ainsi restaurer l'origine grecque de la philosophie, Heidegger ne semble pas très éloigné de la position de son maître Husserl qui pense l'Europe à partir d'un tel héritage pour définir notre tâche dans la période de crise traversée par les peuples européens. Françoise Dastur ${ }^{32}$ insiste à juste titre sur cette convergence de perspectives dans un article consacré à cette question, mais cette convergence, faut-il insister, est en même temps affrontement conflictuel, chacun donnant une signification différente à cette origine.

On peut distinguer quatre divergences qui conduisent à une tout autre interprétation de l'Europe: tout d'abord, Husserl ne distingue pas comme le fait Heidegger un premier commencement aussitôt recouvert et trahi; Heidegger remonte à une source grecque archaïque, nietzschéenne, et oppose le Logos héraclitéen à ce qui va peu à peu se définir comme la raison associée à l'idée de la recherche de la vérité, entendue comme correspondance soumise à la logique; Husserl au contraire, envisage une continuité, malgré certaines différences, entre les différents moments de la rationalité grecque depuis Thalès, puis entre la raison grecque et la raison occidentale moderne. Ensuite, il associe la philosophie à la rigueur démonstrative de la géométrie alors que jamais Heidegger n'évoque cette proximité. En outre Husserl n'établit jamais, comme le fait Heidegger, un lien privilégié entre la Grèce et l'Allemagne: si, selon Heidegger, le destin de l'Allemagne est de répéter l'instauration grecque, Husserl insiste au contraire sur l'universalité de l'ouverture grecque qui inaugure l'infinité d'une histoire avec la possibilité d'un progrès, cet héritage étant commun aux peuples européens en dépit des conflits qui les opposent et vaut même au-delà des frontières

31. M. Heidegger, Correspondance avec Elisabeth Blochmann, p. 279 sq.

32. F. Dastur, «Europa und der "andere Anfang" ", in Europa und die Philosophie, H.-H. Gander (éd.), Francfort, Klostermann, 1993 (trad. fr. dans Revue philosophique de Louvain, 104, 2006, p. 1-22). 
de l'Europe pour tous les peuples occidentaux, en sorte que l'humanité européenne, s'affirme au-delà des nations et même des limites géographiques de l'Europe. Enfin, Heidegger récuse radicalement l'interprétation du thaumazein - étonnement philosophique qui, selon lui, pose la question de l'Être en tant que tel - comme instauration d'une attitude théorique pure. Pour Heidegger, la théorie est un bouleversement radical de l'existence, une modalité de la praxis: «il ne s'agissait pas [pour les Grecs] d'assimiler la praxis à la théorie, mais au contraire de comprendre la théorie comme la plus haute réalisation de la praxis la plus authentique ${ }^{33}$. Alors que, pour Husserl, il est nécessaire de préserver le caractère désintéressé, purement théorique, de la démarche philosophique et scientifique, pour Heidegger un tel geste est caractéristique de l'attitude objectivante qu'il faut refuser. Les oppositions semblent l'emporter sur la convergence.

\section{Les Allemands et les tâches présentes}

La confrontation s'est effectuée publiquement. Heidegger connaissait certainement les idées de Husserl sur le rapport entre philosophie, d'origine grecque, et l'humanité européenne. Inversement, la conférence de Vienne de 1935 est de manière incontestable une réponse au Rektoratsrede de 1933. Ce dernier texte n'est pas seulement un texte politique mais aussi bien un texte philosophique et Heidegger s'y réfère lui-même à de nombreuses reprises, affirmant que «tout s'y trouve ${ }^{34}$. Le Rektoratsrede de 1933 doit être lu selon cet enjeu.

Le titre, Die Selbstbehauptung der deutschen Universität, peut être traduit par: «l'auto-affirmation de l'Université allemande». La philosophie comme science fondamentale y est pensée dans le cadre de l'université, institution qui apparaît comme une communauté de recherche et d'enseignement, ce qui implique la valorisation de la dimension éducative de la science. Cette institution est rapportée au peuple allemand alors que Husserl, quant à lui, n'associe guère la science à l'université, peut-être parce qu'en 1935 il l'a déjà quittée, mais aussi et surtout parce qu'il se fait de l'activité philosophique et scientifique une autre conception que Heidegger. Ce dernier se situe dans la filiation de Schelling ${ }^{35}$ qui pense explicitement l'université comme

33. M. Heidegger, Die Selbstbehauptung der deutschen Universität, in Reden und andere... (GA; 16); L'auto-affirmation de l'université allemande, G. Granel (trad. fr.), Mauvezin, Trans-Europ-Repress, 1982, p. 10.

34. «Dans mon Discours du Rectorat, tout s'y trouve aussi ramassé que possible, comme il convenait en cette occasion", écrit Heidegger dans l'Introduction à la métaphysique, p. 60 .

35. J.-F. Courtine, «Un peuple métaphysique», Revue de métaphysique et de morale, 3, 2003. 
allemande - par opposition à l'universalisme des Lumières et à l'université médiévale qui est européenne et latine. Dans son autobiographie ${ }^{36}$, Jaspers rappellera cette origine transnationale et supra-étatique des universités afin de montrer ce que peut signifier la recherche désintéressée du savoir. Heidegger prononcera quant à lui une conférence, prolongeant le Discours $d u$ Rectorat, dans laquelle il expose la tâche de l'Université allemande ${ }^{37}$ qui naît, précise-t-il, à la fin des guerres napoléoniennes avec la Restauration en Prusse et la fondation de l'Université de Berlin.

L'université est donc un enjeu dans l'existence du peuple allemand, qui dépend d'elle comme elle de lui. On peut d'ailleurs supposer que le titre renvoie à cette relation: le Selbst de l'affirmation n'est sans doute ni le soi de Heidegger ni le soi de l'université mais celui du peuple allemand qui se trouve convoqué à exister de manière authentique. D'ailleurs, dans son cours sur la logique de l'année suivante, Heidegger s'interroge longuement sur ce que nous sommes. Qui sommes-nous nous-mêmes? Cette question renvoie à une décision, et il faut décider de ce que nous sommes en tant que peuple; le Selbst qui pose cette question n'est pas un sujet flottant indéterminé, privé de sol, mais le peuple qui est à lui-même la réponse à la question qu'il pose: «à travers la question "qui sommes-nous?" se décide le destin de celui qui la pose». Le questionneur est impliqué dans sa propre question ${ }^{38}$.

C’est pourquoi la science ne peut pas être désintéressée, «théorique». L'historicité telle que la comprend Heidegger exclut un tel détachement. La science est une modalité de mon existence, c'est une manière d'être du Dasein, et le Dasein est toujours singulier: singularité de l'individu mais aussi du peuple au sein duquel l'individu accomplit son histoire. La science est en crise, elle s'éparpille en une multitude de disciplines particulières et a perdu le sens de sa fondation. Elle se donne alors à elle-même l'illusion d'être une recherche qui vaut par et pour elle-même, mais elle oublie par là sa provenance et, en elle, nous nous oublions nous-mêmes. Si nous nous voulons nous-mêmes, en voulant la science, il nous faut la restaurer dans son authenticité en retrouvant son origine. La science est grecque et ne provient pas de l'ingéniosité de géomètres qui ont inventé la démonstration et une nouvelle méthode de savoir : elle résulte du rapport original du peuple grec - que doit répéter le peuple allemand - à l'étant dans son entier, perspective que ne saurait partager Husserl.

36. K. Jaspers, Autobiographie philosophique, Paris, Aubier, 1963, p. 103.

37. M. Heidegger, Reden und andere... (GA; 16), p. 285 sq.

38. M. Heidegger, Logik als die Frage nach dem Wesen der Sprache, Francfort, Klostermann (GA; 38), 1998; La logique comme question en quête de la pleine essence du langage, F. Bernard (trad. fr.), Paris, Gallimard, 2008, p. 52 sq. 
C'est là [dans l'irruption de la philosophie grecque] que pour la première fois l'homme occidental, à partir du génie d'un peuple et grâce à la langue de ce peuple, se dresse en face de l'étant dans sa totalité, qu'il l'interroge et le saisit comme l'étant qu'il est; toute science est philosophie, qu'elle le sache ou non, qu'elle le veuille ou non. Toute science reste affectée par ce commencement de la philosophie ${ }^{39}$.

Ainsi qu'on le voit, il ne s'agit pas simplement de connaître tel ou tel domaine de la réalité, et Heidegger brocarde «le calme plaisir de s'occuper sans danger à faire avancer simplement le progrès des connaissances ${ }^{40}$. La science apparaît fondamentalement comme une certaine détermination de l'existence d'un peuple historique, dont dépend le sens de notre existence présente. L'oubli de cette provenance conduit à vivre dans l'égarement. Il faut donc «regagner [zurückgewinnen] deux propriétés éminentes de l'essence grecque de la science, les regagner en faveur de notre existence [Dasein] »: être livré au destin et interroger l'étant dans sa totalité. La science redeviendra alors ce qu'elle a été pour les Grecs, «le centre le plus intimement déterminant de l'existence du peuple dans son État». On comprend en quel sens la théorie n'est pas une connaissance désintéressée mais un travail, une certaine manière d'œuvrer ["am Werke sein»], pour tenter de s'accomplir soi-même comme peuple dans le dur combat par lequel celui-ci «maintient à son point d'acuité l'ensemble de son existence».

De cette façon notre existence devient spirituelle [geistig]. La crise de l'Occident est une crise de l'esprit, Heidegger en convient avec Valéry: «La force spirituelle de l'Occident flanche [...] toutes ses jointures craquent ${ }^{41}$. Mais le diagnostic est différent: selon lui, l'esprit se méconnaît, s'est perdu dans l'insouciance en devenant trop «spirituel», quintessencié. Séparé de la vie dans sa facticité et historicité, l'esprit [Geist] est devenu jeu d'esprit [Witz], intellect vide et séparateur réclamant la liberté d'examen sur toute chose - une prétendue liberté académique purement négative, simple licence arbitraire, indéterminée, et pour cela une liberté inauthentique. L'Europe moderne est finalement cette décadence de l'esprit perdu dans le confort et l'insouciance où la science se trouve emportée. L'institution du savoir est corrompue en son principe même, dévoyée dans l'affairement, la curiosité multiforme et désintéressée. Il faut donc non pas tant « retourner aux choses » que retourner les choses, les rétablir, en revenant à la source grecque, présocratique et

\footnotetext{
39. M. Heidegger, L'auto-affirmation..., p. 8.

40. Ibid., p. 11.

41. Ibid., p. 22
} 
en la restaurant. De cette façon, l'Esprit, qui n'est pas Esprit du monde [Weltgeist] mais Esprit du peuple allemand, renaîtra de ses cendres et retrouvera sa grandeur.

Il est alors impératif de se placer sur «le poste le plus avancé du danger que constitue l'incertitude permanente du monde» et se maintenir soi-même dans la grandeur de son peuple, ceci afin de permettre au peuple d'exister dans son véritable monde spirituel:

le monde spirituel d'un peuple ce n'est pas la superstructure d'une culture, ni davantage un arsenal de connaissances et valeurs utilisables, mais la puissance de conservation la plus profonde de ses forces de terre et de sang, en tant que puissances d'émotion la plus intime et puissance d'ébranlement la plus vaste de son existence; seul un monde spirituel garantit au peuple la grandeur.

Ces formules évoquent la célèbre affirmation de Hegel sur l'esprit qui affronte le négatif et se maintient à travers la mort elle-même; cependant, Heidegger ne pense pas, lui, la négativité hégélienne et distingue l'esprit tel qu'il l'entend de la raison du monde [Weltvernunft], qui surmonte la négation et restaure le calice de l'esprit. Pour Heidegger, la révolution est permanente, il n'y a pas de synthèse ou de réconciliation dialectique. Il faut en permanence répondre à "l'incertitude permanente du monde ${ }^{42}$. L'esprit est feu, selon la formule d'Héraclite, il brûle toujours, et la pensée est combat.

Il en résulte que le rôle spirituel de l'université est de combattre. L'homme est l'être des lointains mais le présent le temps de la décision. Il ne s'agit donc pas de préserver l'Allemagne telle qu'elle est empiriquement, aujourd'hui, mais au contraire de l'engager dans son destin. L'Allemagne doit alors, s'enracinant dans l'origine grecque, retrouver cette grandeur qu'elle n'a plus mais qui est pourtant la sienne selon son destin: elle est envoyée vers un futur, que le présent a pour tâche d'accomplir, et qui renouvellera radicalement l'être allemand. L'université est le lieu privilégié de ce renouveau parce qu'elle est le lieu de la vérité et de l'éducation, le lieu d'existence de la philosophie. Depuis ses cours de 1919 (Kriegsnotsemester) ${ }^{43}$, Heidegger ne cesse de penser à cette réforme de l'université. Il ne s'agit pas pour lui de démocratiser l'université, de faciliter le cours des études ou de changer la répartition des disciplines, mais de refonder la science dans sa totalité à partir de la philosophie conçue comme ontologie radicale et de restaurer le Dasein dans une existence authentique:

42. M. Heidegger, L'auto-affirmation...

43. M. Heidegger, Zur Bestimmung der Philosophie, Francfort, Klostermann (GA; 56-57), 1987. 
La désintégration en disciplines est la fin de l'université, laquelle est déjà là depuis des décennies, parce que depuis longtemps une force éducative unitaire fait défaut. Est-ce qu'il n'y a plus de pouvoir créateur unitaire, est-ce que la force au cœur du peuple allemand ne sait plus se maintenir fermement soimême, mais seulement se cramponner à ce qui a été jusqu'ici ${ }^{44}$ ?

Et Heidegger déplore que «ceux qui révolutionnent ne remarquent pas que nous ne conservons qu'un cadavre, une unité de façade ${ }^{45}$. Il écrit à Jaspers: «Nous travaillons [...] à la réanimation de la philosophie » ${ }^{46}$;

Ou bien nous nous mettons sérieusement à la philosophie et à ses possibilités comme recherche scientifique principale, ou bien nous consentons en tant que scientifiques au manquement le plus grave, celui de barboter dans des concepts repassés et des partis pris à moitiés clairs et de travailler à la commande. Si le premier cas est adopté, on aura choisi le danger de mettre en jeu son existence extérieure et intérieure tout entière pour quelque chose dont on ne pourra voir soi-même le résultat ni l'issue ${ }^{47}$.

La philosophie est " quelque chose d'entier et d'extrême » ${ }^{48}$, sur elle repose la totalité de cette organisation du savoir et la confrontation avec l'extrême est le plus dangereux: la philosophie est le contraire de tout apaisement et de toute assurance. Elle est le tourbillon dans lequel l'homme est entraîné vertigineusement, jusqu'à comprendre le Dasein à partir de lui seul, en renonçant à toute «fantasmagorie». Réformer l'université consiste à faire cesser cette "fantasmagorie» que sont devenues la science et la culture européennes aujourd'hui. Il revient à l'université allemande d'accomplir cette tâche et par conséquent de sauver l'Europe. Comme le dira Heidegger à Rome en 1936 dans sa conférence sur «l'Europe et la philosophie allemande», "notre être [Dasein] historique fait l'expérience avec une détresse croissante et avec la plus grande clarté que son futur peut également conduire à l'une ou l'autre de ces deux possibilités élémentaires: ou bien l'Europe sera sauvée ou elle sera détruite !» 49 ; «L'histoire de l'esprit allemand est le destin du peuple allemand [...]. Ainsi l'histoire de l'université allemande est l'histoire du XIX ${ }^{\mathrm{e}}$ siècle ${ }^{50}$.

44. M. Heidegger, La logique comme question..., p. 93.

45. Ibid.

46. M. Heidegger, Correspondance avec Karl Jaspers, Paris, Gallimard, 1996, p. 11. Lettre du 21 mars 1920.

47. Ibid., p. 23 et 24 . Lettre du 27 juin 1922.

48. M. Heidegger, Concepts fondamentaux..., p. 20.

49. Europa und die Philosophie, p. 31 (nous traduisons).

50. M. Heidegger, Die deutsche Universität, in Reden und andere... (GA; 16), p. 285 (nous traduisons). 


\section{L'Allemagne et le milieu}

L'université allemande se voit reconnaître un rôle insigne. Elle est l'histoire: non pas le lieu de l'Historie, histoire des historiens, mais de l'être historique [Geschichtlichkeit]. Cette histoire sédimentée en elle nous détermine à notre insu, elle n'est pas une histoire de ceci ou de cela mais histoire de l'Être. Elle est constitutive de notre rapport à la vérité. Nous sommes toujours déjà dans un rapport à la vérité, même si nous ne le savons pas, même si nous l'avons "oublié»; et en un sens cet oubli est inévitable puisque nous sommes pris dans la préoccupation de la vie quotidienne et l'illusion de l'évidence des objets de notre affairement. Mais la tâche s'impose de faire retour sur cette situation afin de saisir ce qui nous est envoyé, notre destin [Geschiklichkeit]: dans Sein und Zeit, Heidegger analysant cette notion de destin distingue la destinée individuelle du destin collectif et établit que l'individu existe certes, mais dans et à travers une communauté, une des composantes essentielles du destin étant l'appartenance à une génération.

Or cette notion de génération est explicitée dans un texte de 1934 intitulé 25 ans après notre baccalauréat. Ce texte contient, à nouveau, une récusation de la philosophie du contrat social. Une génération, explique-t-il, reliant des camarades en un lien qui ne saurait être pensé comme l'effet d'un contrat calculateur passé entre individus; et c'est au contraire une Gemeinschaft et une Gefolgschaft originaires qui résultent du partage d'un destin. Ce destin suppose la confrontation avec la mort de nos camarades tombés à la guerre pour défendre notre peuple. Certes, ces camarades sont morts et l'Allemagne a été vaincue mais, dit Heidegger, la défaite n'a rien tranché, ni pour les vaincus ni pour les vainqueurs, la fin des hostilités et le traité de Paix ne décidant rien. Et la défaite, pourrait-on dire, nous place même devant une décision encore plus redoutable que celle à laquelle la victoire nous aurait confrontés, la décision de savoir ce que nous sommes en tant que peuple affecté par ce qui arrive, das ungeheure Geschehen. La défaite nous renvoie vers notre futur le plus lointain et notre capacité de l'accomplir. Il faut en effet distinguer deux sortes de peuple, ceux qui vieillissent et ceux qui s'éveillent: la décision se trouve dans cette alternative et non dans la défaite ou la victoire. Sommes-nous un peuple qui s'éveille? Si tel est le cas, la guerre nous invite à nous retrouver nous-mêmes, à nous affirmer dans l'authenticité de notre être, dans la plénitude de notre monde spirituel et de ses soubassements, à travers la lutte contre le processus historique de la chute. Reprenant un vocabulaire nietzschéen, Heidegger affirme que tel est bien le cas: "Nous sommes ceux qui sont un dépassement et un pont»: «Unser Geschlecht ist der Übergang und die Brücke [...] ist die Brücke zur 
geistigen geschichtlichen Eroberung des grossen Krieges " ${ }^{51}$. La guerre est un combat spirituel et la défaite nous provoque, nous projette vers le futur spirituel de notre peuple qui se révèle, par là, le peuple du futur par excellence, rejetant définitivement dans le passé révolu [Vergangenheit] toute la pseudo-pensée qui réduisait notre être à son apparence [Scheinwelt], «le méli-mélo habituel provenant d'un humanisme inauthentique». Il faut couper les branches mortes! L'esprit est combat et le combat permet de distinguer, départager le vivant du mort, le passé du futur, le spirituel de ce qui va se perdre dans l'insignifiant et l'inessentiel. Nous retrouvons la sentence héraclitéenne sur le combat père de toutes choses. Comprenons que c'est sans doute par sa parenté spirituelle avec la Grèce que l'Allemagne s'affirme dans sa vérité à travers le combat. Ainsi l'Allemagne, combattant pour elle-même, combat aussi pour l'esprit, c'est-à-dire pour la préservation de l'Occident contre l'Asiatique! Comment faire apparaître cette élection de l'Allemagne?

Notre génération est au milieu! Elle est, d'abord au présent, dans l'Augenblick entre passé et futur. Le passé n'est ainsi pas simplement le révolu qu'il faut définitivement faire passer, il est aussi das Gewesene, ce qui, devenu et persistant au fond de nous, constitue notre être même, la plupart du temps à notre insu. En ce sens, notre passé, c'est la Grèce. Non pas n'importe quelle Grèce. Car il y a une Grèce «classique», celle des Lycées allemands, celle véhiculée par l'humanisme, objet du travail des philologues et des éloges enflammés mais bien pensants: cette image de la Grèce doit elle aussi être déconstruite en tant qu'elle est un mode de l'oubli de la Grèce authentique. Il faut apprendre à lire et redécouvrir, comme Nietzsche ou Hölderlin, le premier commencement, ouverture de notre histoire. Nous sommes au milieu entre le passé grec et l'avenir du peuple allemand. La pensée est souvenir [Gedank] et fidélité ${ }^{2}$.

Mais l'histoire est aussi celle de l'Occident et le peuple allemand occupe ici encore le milieu. Les Allemands sont, pour Heidegger, le peuple historique par excellence, celui qui s'éveille ébranlé par la guerre, ouvert sur l'avenir, un peuple qui est un pont, permettant le passage vers le renouveau spirituel de l'Occident dans son entier. Mais un peuple est institué par sa langue, ses poètes et ses penseurs, et l'allemande est la plus à même d'établir un rapport authentique avec les Grecs. Entre l'Allemagne et la Grèce il existe une affinité qui se marque par la possibilité propre à l'allemand de traduire les paroles originaires des Grecs. Ce n'est certes pas la langue

51. M. Heidegger, 25 Jahre nach unserem Abiturium, in Reden und andere... (GA; 16), p. 279 sq.

52. M. Heidegger, Qu'appelle-t-on penser?, Paris, PUF, 1959, p. 152; Was heisst Denken?, Tübingen, Niemeyer, 1961 [cours de 1951], p. 96. 
comme telle qui traduit mais la langue germanique contient bien cette possibilité dont l'accomplissement dépend, estime Heidegger, de notre engagement résolu à répéter la parole grecque dans son originarité. L'histoire est mythologie ${ }^{53}$. La langue n'est pas soumise à la logique - logique universelle qui déterminerait la structure du logos, de la raison commune à tous les hommes. Il faut penser tout à l'inverse le logos comme langue et la langue comme configuration d'un monde par ces héros de la pensée de l'Être que sont les poètes et les penseurs:

la langue en tant qu'à chaque fois historique, n'est rien d'autre que l'événement dans le tout de l'étant de l'exposabilité transmise par l'Être [...]. La langue est ce qui gouverne le centre à partir duquel le monde de l'existence historique du peuple se configure et se préserve... La langue originale est la langue de la poésie ${ }^{54}$.

La parole grecque originaire, celle des "philosophes" présocratiques institue le monde occidental comme tel, elle éclaire les étants à partir de la lumière de l'Être. Les mots phusis, aletheia, les fragments d'Héraclite, de Parménide, mais aussi les strophes de Sophocle, ouvrent notre monde en disant originairement ce qui est. La tâche est de restituer cette parole, la traduire et la commenter, en détruisant les obstacles - les mauvaises traductions sédimentées dans la culture européenne et les humanités allemandes - à sa compréhension authentique. La langue par excellence de cette traduction est l'allemand, qui trouve aussi bien à se régénérer dans sa fidélité à la Grèce:

Là où, par conséquent, il est nécessaire de porter à l'écoute la parole grecque antique, la traduction peut suffire, à condition bien sûr que l'élucidation de ce que nous dit cette parole ne fasse pas défaut et que celle-ci soit pensée à fond dans l'horizon de notre expérience et de notre savoir propre. D'ailleurs, la langue des Allemands est propre comme nulle autre langue à traduire la parole grecque antique, et ce éminemment si la parole grecque n'est pas transposée dans un simple usage présent de la langue allemande mais si ce dernier luimême trouve du même coup à s'y renouveler et régénérer ${ }^{55}$.

Ou encore: «La langue grecque est, avec l'allemande, du point de vue des possibilités du penser, à la fois la plus puissante de toutes et celle qui est le plus la langue de l'esprit ${ }^{56}$.

53. M. Heidegger, Introduction à la métaphysique, p. 162.

54. M. Heidegger, La logique comme question..., p. 199 (nous modifions la traduction selon le texte allemand Logik als die Frage... (GA; 38), p. 168-170).

55. M. Heidegger, Concepts fondamentaux, Paris, Gallimard, 1985, p. 31; Grundbegriffe, Francfort, Klostermann (GA; 51), 1981.

56. M. Heidegger, Introduction à la métaphysique, p. 67. 
Le milieu, c'est donc aussi l'alternative entre la persistance possible de la chute et la régénérescence. Or de ce point de vue la pression qui s'exerce sur l'Allemagne est extrême. L'Allemagne se trouve, en effet, au milieu de l'Occident et de l'Europe envisagée comme continent. Elle est prise comme dans un étau entre l'Est et l'Ouest et devient alors cette partie de l'Europe qui subit la crise avec le plus d'acuité, portant aussi par conséquent la responsabilité essentielle. La crise semble venir des deux côtés à la fois: d'abord par l'Est et la confrontation avec le communisme - non pas avec les Russes qui, selon le Heidegger lecteur enthousiaste de Dostö̈evski, sont «spirituels» - mais avec le communisme, c'est-à-dire: «les Soviets + l'électricité", les masses et la technique moderne; mais aussi bien par l'Ouest où nous trouvons les Anglais et surtout l'Amérique, c'est-à-dire la démocratie et le progrès technique. Dans les deux, l'esprit est en péril. Car l'Est et l'Ouest reviennent essentiellement au même et ce même est sans doute ce que vise Heidegger avec le nom d'Asiatique, c'est-à-dire la puissance du gigantesque et de l'indistinction, le règne de la grandeur simplement quantitative qui refuse le rang et la singularité combattante de chaque peuple ${ }^{57}$. Les Grecs ont affronté la démesure orientale, nous devons, quant à nous, affronter la démesure moderne, à savoir l'égalisation générale qui fait proliférer l'indifférent et réduit l'esprit à l'intellect ou à la raison calculante, ce qui rend l'esprit impuissant, défait - Heidegger parle de l' «Entmachtung des Geistes» - et prépare «le règne des littérateurs et des esthètes ${ }^{58}$. De ce point de vue la Cité grecque n'est pas ce que l'on croit, un espace où le peuple, demos, délibère dans l'isagoria et l'isonomia, mais est au contraire le site à partir duquel le peuple, ethnos, est institué par ses poètes, penseurs et hommes d'État, des êtres de haut rang, des héros. L'Être est logos, ce qui ne veut pas dire raison, cette dernière renvoyant à la ratio latine ou cartésienne commune à tous les hommes et qui rend possible le débat contradictoire dans la cité démocratique ${ }^{59}$, conception " anodine et sentimentale» qui fait dépendre la décision de compromis ou de calculs; la raison est méconnaissance du vrai sens du logos qui veut dire legein, rassembler. Le logos est recueillement originaire, or,

c'est parce que l'être est, en tant que logos, recueillement originaire et non pas un fourre-tout, un mélange où tout aurait autant et aussi peu de valeur, que la grandeur et la souveraineté appartiennent à l'Être. Si l'Être doit être patent

57. Ibid., p. 57.

58. Ibid., p. 58.

59. «La démocratie est uniquement, comme Nietzsche l’a clairement vu, une forme dégénérée de nihilisme» (M. Heidegger, Nietzsche. Der Wille zur Macht als Kunst, Francfort, Klostermann (GA; 43), 1985, p. 193). 
il faut qu'il ait et garde son rang. Héraclite, en parlant de la multitude comme de chiens et d'ânes, exprime cette attitude. Elle appartient essentiellement au Dasein grec. Puisque l'on se réfère parfois aujourd'hui à la cité grecque avec un zèle excessif, on ne devrait pas passer sous silence cet aspect, sans quoi l'on risquerait de se faire une idée anodine et sentimentale de la polis. Ce qui a rang est quelque chose de fort ${ }^{60}$.

L'esprit est donc combat, refus du compromis qui, supprimant les tensions, conduit au nivellement; il est respect du rang. Seuls ceux qui acceptent la lutte et en sortent vainqueurs accèdent à la vérité. Telle est la leçon d'Héraclite.

L'Allemagne est donc au milieu de deux puissances d'indistinction.

Cette Europe qui dans un incurable aveuglement se trouve sur le point de se poignarder elle-même est prise dans un étau entre la Russie d'un côté et l'Amérique de l'autre. La Russie et l'Amérique sont toutes deux, du point de vue métaphysique, la même chose; la même frénésie sinistre de la technique déchaînée et de l'organisation sans racine de l'homme normalisé ${ }^{61}$.

L'Europe apparaît ici comme le centre qui résiste encore à cette attaque contre l'esprit. L' "Entmachtung des Geistes» ayant vaincu la terre tout entière revient sur son centre par l'Est et l'Ouest pour achever son œuvre.

La décadence spirituelle de la terre est déjà si avancée que les peuples sont menacés de perdre la dernière force spirituelle qui leur permettrait du moins de voir et d'estimer comme telle cette décadence conçue dans sa relation au destin de l'Être ${ }^{62}$.

Et au centre de l'Europe [in der Mitte] se trouve le peuple allemand qui est alors le plus en danger, et de ce fait le plus décisif, celui dont dépend l'issue de la crise.

Nous sommes pris dans l'étau. Notre peuple, en tant qu'il se trouve au milieu, subit la pression la plus violente, mais lui qui est le peuple le plus riche en voisins est aussi le plus en danger et le peuple métaphysique ${ }^{63}$.

Ce peuple doit donc accepter le destin qui est le sien, s'exposer à l'intérieur du combat spirituel fondamental. C'est la seule possibilité pour que l'Occident, tel qu'il a été institué par les Grecs comme le séjour de l'esprit, puisse subsister et se projeter comme tel dans l'avenir. Ce destin de l'Occident

60. M. Heidegger, Introduction à la métaphysique, p. 141 (trad. légèrement modifiée).

61. Ibid., p. 49 .

62. Ibid., p. 49.

63. Ibid., p. 49. 
peut ne pas s'accomplir et l'Occident sombrer dans l'anéantissement de l'oubli radical de l'Esprit, mais l'Occident peut aussi se maintenir comme Occident en préservant le sens de l'Être. La «grande décision» dépend du peuple allemand:

si l'on ne veut pas que la grande décision concernant l'Europe se produise sur le chemin de l'anéantissement, c'est précisément par le déploiement de nouvelles forces spirituelles en tant qu'historiques issues de ce centre qu'elle doit se produire.

L'Europe est ici le lieu d'un combat pour l'esprit de l'Occident ${ }^{64}$, combat qu'il appartient au peuple allemand de mener afin de le sauver et, avec lui, la terre tout entière. L'Allemagne est au centre de la terre, au centre de l'histoire pensée comme histoire de l'Être. Notre peuple, estime Heidegger, doit alors entrer en résonance [Widerhall] avec sa destination et cette résonance est répétition [Wiederholung]. Répéter n'est pas alors revenir en arrière vers un passé objectivé, mais recommencer plus originairement dans l'obscurité et le trouble du premier combat.

Ainsi nous comprenons que la crise vient de l'Europe elle-même et Europe pourrait même être un des noms de cette crise. "L'énervation [Entmachtung] de l'esprit vient de lui-même», elle a contaminé jusqu'à l'Allemagne et l'idéalisme allemand qui s'est effondré. Mais la grandeur de cet idéalisme, "du peuple des penseurs et des poètes », n’est pas en cause: un processus au très long cours a produit ses résultats décisifs au début du XIX ${ }^{\mathrm{e}}$ siècle et a rendu impossible le maintien de cette grandeur en désintégrant progressivement toutes les forces spirituelles de l'Europe. Peu à peu toutes choses sont devenues égales, indifférentes, distinguées simplement par le nombre de telle sorte que la grandeur et la distinction de l'existence singulière se sont trouvées systématiquement détruites. Cette indistinction nihiliste s'est étendue aux extrêmes du monde occidental et s'attaque désormais à nouveau à son cœur dans un combat décisif et sans doute ultime. L'esprit est confronté à la démonie [das Dämonische], ce que Nietzsche appelle «die Rache» (la méchanceté destructrice: «im Sinne des zerstörerisch Bösartigen»). Il se doit de restaurer hiérarchie, rang, singularité, sens de la grandeur. "L'esprit est le plein pouvoir donné à l'étant comme tel en totalité, là où l'esprit règne, l'étant comme tel devient toujours et en toute occasion plus étant ${ }^{65}$. Tel est vraisemblablement le sens de la participation de Heidegger au mouvement de la "révolution allemande» dont il célébrera, encore après la guerre, la grandeur. Restaurer l'Allemagne

64. Ibid., p. 51 (Einführung in die Metaphysik, p. 30).

65. Ibid., p. 60. 
comme puissance spirituelle au centre de l'Europe pour sauver l'Occident, fonder à nouveau l'Occident comme tel, non pas seulement dans l'esprit de Platon à Syracuse mais aussi d'Héraclite à Éphèse, telle est la tâche.

Pendant le «mouvement», l'objectif principal de Heidegger était de maintenir la radicalité révolutionnaire en luttant contre l'emprise de "l'organisation", de la préoccupation utilitariste et scientiste qui faisait retomber la flamme de l'Esprit à l'intérieur de la sphère des préoccupations ontico-mondaines. Le Mouvement devait être radicalisé et révolutionné pour échapper à l'emprise du Verfall.

\section{L'Europe comme chute}

Heidegger veut donc provoquer l'élan qui permettra de répéter l'origine de notre histoire. La crise de l'Occident est la conséquence d'un oubli dont les effets font retour sur le centre à partir des extrémités, prenant la forme d'un destin. Comment définir cette chute? «Seul le philistin et le journaliste ne voient qu'arbitraire et violence dans le fait de déterminer le sens des mots ${ }^{66}$. La chute va apparaitre dans le processus de la reprise des mots et des paroles fondamentales des Grecs. Notre "métaphysique» est « un langage que nous parlons tous, que l'Europe parle, mais vingt fois transposé, mais verni, aplati, usé et sans arrière-fond ${ }^{67}$.

L'instauration grecque est aussitôt recouverte: Platon «reprend» ses prédécesseurs, mais les altère et nous éloigne de la lumière de l'Anfang, de la prise initiale de l'être. Platon n'est plus examiné à partir d'Aristote, mais d'Héraclite: c'est la première chute, le premier oubli. Cet égarement platonicien prépare toute une série d'étapes qui s'enchaînent et s'accumulent. Elles constituent une histoire de l'Europe, laquelle n'est plus un simple synonyme de l'Occident:

Nous questionnons pour savoir quel est l'appel inexprimé qui renvoie au commencement de la pensée occidentale, sur les traces de laquelle nous pensons aussi, nous modernes, même si entre-temps l'occidental a sombré dans l'européen ${ }^{68}$.

L'Europe peut alors se définir comme: la raison, l'humanisme, les lumières, les sciences physico-mathématiques, les droits de l'homme, la démocratie, c'est-à-dire l'oubli de l'Occident. Certes, très souvent occidental et européen sont synonymes, ayant même provenance, l'Occident inauguré

66. M. Heidegger, La logique comme question..., p. 152.

67. M. Heidegger, Qu'appelle-t-on penser?, p. 117.

68. Ibid., p. 172. 
par la parole grecque, mais européen signifie souvent l'occidental dans sa chute, ce Verfall, qui risque de nous perdre, nous détournant de nousmêmes.

Le recouvrement platonicien en prépare un autre: Platon introduit une scission, chorismos, entre "l'étant purement apparent ici-bas et l'être réel quelque part là-haut", dans laquelle s'installera plus tard la doctrine du christianisme. Certes, cette doctrine introduira en outre un changement de perspective en interprétant l'inférieur comme créé et le supérieur comme créateur, mais c'est avec les armes de l'Antiquité ainsi refondue que le christianisme se posera en s'opposant à elle, conçue comme paganisme ${ }^{69}$. Ainsi, le christianisme approfondit l'occultation d'un héritage et doit être, comme tel, déconstruit: il dépend de la source grecque qu'il transmet en retournant complètement sa signification. Déconstruire signifie toujours défaire, décomposer les éléments pour les recomposer selon leur véritable signification, et tenter de restaurer l'originaire en faisant disparaître ce qui l'occulte. L'originaire n'est désormais plus, comme en 1920, l'existence chrétienne primitive, mais la parole d'Héraclite.

Le christianisme dépend aussi d'une autre opération. Il n'est pas seulement platonicien, mais également d'origine juive, et dépend alors d'une interprétation juive de l'héritage grec. Certes les apôtres écrivent en grec, mais une des paroles originaires de la philosophie grecque, le Logos héraclitéen, a été subtilement détournée de son sens: alors qu'il signifie originairement l'Être de l'étant, le recueillement rassemblant les étants dans l'unité de l'Être, la récollection des forces antagonistes, il renvoie maintenant à un étant particulier et perd sa portée ontologique pour se dégrader dans l'ontique. Le christianisme est oubli de l'Être et ce, dans la parole même de l'Être. Le mot Logos va désigner le fils de Dieu, « et celui-ci dans sa fonction de médiateur entre Dieu et l'homme. Cette représentation néo-testamentaire du Logos est celle de la philosophie religieuse juive que Philon a élaborée ${ }^{70}$. Logos devient le nom du commandement, de l'ordre, ordre donné à partir de la croix, par un crucifié! «Un monde sépare tout cela d'Héraclite», nous dit Heidegger. Certes! Le christianisme apparaît comme un judéo-christianisme, marqué par la croix - châtiment réservé aux esclaves - devenue symbole du sauveur. La "parole" prêche donc désormais obéissance et soumission à la loi de l'Ancien Testament et promet, du haut de la croix, la résurrection des corps, invitant à fuir ce monde, trahissant la temporalité de l'être en évoquant une éternité imaginaire. Le

69. M. Heidegger, Introduction à la métaphysique, p. 114.

70. Ibid., p. 142. 
christianisme est une morale d'esclaves, un ressentiment contre le devenir, une "métaphysique», un nihilisme, une «méchanceté destructrice»! Telle est «l'Europe».

L'Europe est donc équivoque. Instituée dans la lumière de l'Être et s'éloignant de l'Être qui l'institue. Ou peut-être secrètement instituée par ce qui se montre en se dissimulant pour se dissimuler dans son retrait: l'Être est absent dans sa présence, tout est figure, interprétation, et « figure porte absence et présence », disait Pascal. Le christianisme est de ce fait une figure multiforme: il est un figuratif du judaïsme, comme il vient d'être vu, mais aussi du monde scientifique moderne, de la démocratie, du communisme et de son contraire, l'américanisme. Heidegger joue en permanence sur ces renversements: le bolchevisme peut apparaître comme le danger par excellence et l'Allemagne le dernier rempart de l'Europe contre lui, mais il n'est rien d'autre que l'Europe elle-même portée à son terme et ne fait que déployer jusqu'à ses ultimes conséquences ce qui déjà était contenu dans le christianisme:

Le bolchevisme est originairement occidental, une possibilité européenne: la survenue des masses, de l'industrie, de la technique, du dépérissement du christianisme; aussi loin que la domination de la raison comme égalisation de tous n'est que la conséquence du christianisme et que celui-ci en son fond est d'origine juive (cf. la pensée nietzschéenne de la provenance esclavagiste de la morale), alors le bolchevisme est en fait juif mais le christianisme est fondamentalement bolcheviste! Et quelles décisions deviennent nécessaires à partir de là ${ }^{71}$ ?

Le monde d'Héraclite est tout autre [eine andere Welt]. Voilà les termes du choix.

Mais les avatars du logos ne s'achèvent pas là. Le christianisme est également Rome, et la chute inaugurée par Philon va se conjuguer à une autre. La transmission de la philosophie grecque aux Romains étant en même temps trahison, comme l'avait déjà été le développement de la culture alexandrine. "Traduttore traditore», selon la formule italienne. En effet, Heidegger pense Rome à travers ses institutions, son droit, la République, l'Empire surtout, lequel détermine la vérité comme ordre, mais un traitement spécial est réservé à sa langue. Le latin, langue européenne par excellence, est celle de l'Empire. La répugnance heideggerienne à l'égard de

71. M. Heidegger, Beiträge zur Philosophie, Francfort, Klostermann (GA; 65), 1989, p. 54 (nous traduisons): «sofern aber die Vernunftherrschaft als Gleichsetzung aller nur die Folge des Christentums ist und dieses im Grunde jüdischen Ursprung, [...] ist der Bolschewismus in der Tat jüdisch; aber dann ist auch das Christentum im Grunde bolschewistisch!». 
Rome exprime peut-être un rejet de cet empire universel existant à travers une langue unique qui sera longtemps la langue universelle des savants et de l'Université européenne comme de l'Église chrétienne catholique romaine. Elle est en outre la première langue de réflexion de Heidegger, puisque celle de la scolastique où il s'initia à la pensée.

Le « romain» transmet en trahissant: traduire en «latin-romain » c'est occulter le sens du mot grec. On peut noter que Heidegger ne dit jamais latin mais romain ou latin-romain, sans doute pour accentuer le rapport à «la romanité», aux langues romanes faibles et inauthentiques (et on peut évoquer ici les textes de Fichte par exemple sur cette question) mais aussi à Rome comme centre de l'Église romaine dont l'Allemagne s'est émancipée avec Luther. La langue romaine métamorphose tout et fait perdre aux mots grecs leur dimension ontologique originaire; par exemple humanitas, substantia, religio, ratio; à chaque fois la traduction cache et détourne. Dans Qu'appelle-t-on penser ?72 la raison est analysée à partir de la traduction du logos grec par le latin ratio et avec l'avènement de la ratio tout se renverse. La ratio nous conduit à rechercher l'essence de la vérité du côté de la logique par laquelle on tente alors vainement de s'emparer de l'essence du logos et du vrai. De la ratio on dérive vers le rationalisme, dans son caractère moderne, et cette dérive nous masque la signification de ce que nous appelons toujours le mythe.

La philosophie médiévale et moderne poursuit ce mouvement, et Descartes joue ici un rôle décisif. Il commence par un doute radical, recherche un fondement apparemment incontestable, mais établit le règne de la subjectivité et va rendre possible l'institution des sciences mathématiques de la nature. Celles-ci ne constituent pas un progrès dans la conquête de la vérité à partir de la fondation grecque de la science. En effet la notion même de progrès est radicalement récusée ${ }^{73}$ : elle supposerait que les Grecs soient juste un peu supérieurs aux Hottentots et que nous leur soyons bien supérieurs; alors notre concept de la vérité serait homogène au leur et la science moderne pourrait être comparée à la science grecque. Or, tel n'est pas le cas: la vérité est historique, il n'y a pas une norme de vérité extratemporelle; la vérité est interprétation, elle est éclaircie de l'Être, d'un Être qui s'historicise nécessairement: Galilée n'est pas plus vrai qu'Aristote, pas plus que Shakespeare n'est supérieur à Eschyle. La science moderne n'est pas la science médiévale ni la science grecque. Il y a, chaque fois, une mutation du Dasein dont le principe se trouve dans une Grundstimmung épochale et populaire. La science grecque n'est pas moins vraie que la science

72. M. Heidegger, Qu'appelle-t-on penser?, p. 195.

73. M. Heidegger, Chemins qui ne mènent nulle part, Paris, Gallimard, 1962, p. 71. 
moderne, elle est vraie autrement et paradoxalement plus originaire; elle est vraie ontologiquement parce qu'elle permet de penser l'histoire même de l'Être. On comprend qu'il n'y a pas de cogito heideggerien parce qu'il y a une racine grecque, institution, frappe [Prägung] qui nous invite au recueillement méditatif [Wiederholung] et résonance [Widerklang].

À partir du tournant cartésien, l'Être va devenir l'étant disponible et la raison la faculté subjective dont il faut effectuer la critique pour garantir la vérité des représentations. Et de cette philosophie du sujet va sortir le libéralisme qui méconnaît la nature du peuple en son destin historique; critiquer le libéralisme c'est répéter l'origine, c'est remonter de la mauvaise répétition à la bonne.

C'est dans cette conception de l'homme (de la primauté accordée au je comme sujet) que le libéralisme a sa racine. La bataille contre le libéralisme se cantonne dans la phraséologie usée jusqu'à la corde au lieu de se porter à une authentique révolution de l'être et du savoir dans leur ensemble... mais la manière dont nous faisons émerger l'être de l'homme évite de poser au départ l'homme comme un sujet séparé et elle a pour but de permettre une expérience originalement neuve de l'être de l'homme ${ }^{74}$.

Remonter en deçà de Descartes, penser l'origine c'est déconstruire «le sujet", détruire le libéralisme, exister comme peuple et réciproquement.

La dérive suprême se trouve alors dans la philosophie de l'Aufklärung prolongeant le doute cartésien. D'ailleurs, la philosophie ne doit surtout pas commencer par le doute!

L'Aufklärung a assombri l'origine de l'être de la pensée. Elle barre tous les chemins qui mènent à la pensée des Grecs absolument. Ce qui ne veut pas dire que la philosophie post-hellénique soit fausse ni qu'elle soit une fausse piste mais tout au plus que la philosophie, malgré toute la logique et la dialectique, n'est pas arrivée à entrer dans la question «qu'appelle-t-on penser?». De cette question cachée la philosophie s'éloigne le plus lorsqu'il lui est suggéré que la pensée doit commencer avec le doute ${ }^{75}$.

Cette déconstruction de l'Europe, suivant le fil de «l'étymologie», se retrouve à nouveau dans la Lettre sur l'humanisme, ou bien encore dans l'examen de la notion de religion: l'humanisme vient de l'humanitas latine qui nous éloigne de la relation authentique du Dasein à l'Être. Hölderlin parle des Dieux mais n'est pas religieux, parce que religion vient du latin et trahit le vrai rapport aux dieux. De manière constante le rapport aux Grecs,

74. M. Heidegger, La logique comme question..., p. 176.

75. M. Heidegger, Qu'appelle-t-on penser?, p. 195. 
médiatisé par le vocabulaire latin, est présenté comme fidélité trahie qui témoigne cependant de la source jusque dans cette trahison; cette infidélité, d'ailleurs, n'advient pas de manière quelconque, elle suit un enchaînement, elle est provenance déterminante et en ce sens destin. L'Europe est histoire de l'Être ou déterminée par l'Être, mais c'est aussi une histoire de l'oubli progressif de ce qui l'institue. Même ceux qui, comme Nietzsche, croient pouvoir échapper à cette nécessité en la retournant restent pris en elle, la poussent jusqu'à ses conséquences extrêmes et son achèvement. Le risque suprême étant alors que la révolution allemande soit également surmontée par ce dispositif, et qu'elle aussi échoue.

\section{Le peuple et la Stimmung}

La pluralité des époques nous renvoie à une autre pluralité, celle des peuples. Comme chez Herder, chaque peuple a son esprit propre [" seine eigene Stimmung»], l'accord fondamental de tous ses sentiments les uns avec les autres. Chaque tonalité est à elle-même sa mesure propre. Sur ce point il est clair que Heidegger n'est pas raciste. Il ne définit pas le peuple comme communauté de race biologiquement déterminée. Le racisme suppose la biologie qui doit être elle-même fondée philosophiquement dans l'existence historique du peuple. Il dépend donc du peuple de déterminer, historiquement, ce qui est sain et malsain; tout ce qui est naturel est historique, la santé est historique: "was gesund und krank ist, dafür gibt sich ein Volk und ein Zeitalter je nach der inneren Grösse und Weite seines Daseins selbst das Gesetzt ${ }^{76}$. Le racisme biologique repose sur l'oubli de la différence ontologique. Or la singularité du peuple allemand est ontologique. Elle résulte d'un destin, elle suppose une certaine consonance des éléments de ce peuple entre eux, une consonance avec son sol et elle s'institue à partir d'une langue «frappée » par les fondateurs, poètes, penseurs, hommes d'État. Heidegger développe des éléments de cette analyse, dans son cours sur la Logique de 1934, et l'année suivante dans sa conférence sur L'Origine de l'ouvre d'art.

Il expose également sa conception des rapports entre les peuples dans différents textes prononcés ou écrits à cette même époque. Ainsi, dans le discours qu'il adresse aux étudiants pour appeler au plébiscite en faveur de la décision d'Hitler de quitter la Société des Nations (SDN), il justifie la sortie de l'Allemagne de la SDN, un organisme international inspiré du projet kantien de paix universelle, en affirmant que chaque peuple doit

76. M. Heidegger, Reden und andere... (GA; 16), p. 151. 
suivre sa voie propre. La Société des Nations vient limiter la capacité de décision du peuple allemand, empêche celui-ci de suivre jusqu'au bout sa destinée, d'accomplir sa mission qui est, en même temps, une mission pour tous les peuples. Le peuple allemand doit, ainsi, s'autodéterminer. Il sera alors lui-même et, à travers son combat il remplira sa mission, qui résulte de son privilège ontologique: sauver l'Occident:

Il ne s'agit nullement de nous détourner de la communauté des peuples... la volonté qui veut une vraie communauté des peuples se tient aussi loin d'une fraternisation universelle sans consistance ni engagement véritable que d'une aveugle domination par la violence: elle crée l'ouverture et la vaillance au sein desquelles les peuples et les États peuvent se tenir tant relativement à soi que relativement aux autres ${ }^{77}$.

La politique est combat et non pas négociation stérile et égalisante ${ }^{78}$.

Ce texte peut donner le sentiment que Heidegger présuppose une sorte d'harmonie préétablie entre les peuples, comme un libéralisme international; la même impression se dégage d'une intervention écrite pour un congrès philosophique à Paris, auquel il n'a pas assisté, Wege zur Aussprache, traduite en français sous le titre Chemins d'explication ${ }^{79}$. Il examine la confrontation entre les peuples européens et particulièrement, ici, entre les peuples français et allemand en relation «à la grave menace que subit l'Occident »; il s'agit de sauver l'Occident faute de quoi nous risquerions de sombrer dans un chaos généralisé et d'être complètement déracinés. Or cela suppose que chaque peuple ose être soi-même, il faut refuser une entente factice; l'entente authentique résulte de la confrontation des peuples qui sont fiers d'être eux-mêmes. La fierté résulte de la décision d'être soi-même; ce qui implique de se maintenir à son propre rang. Chaque peuple a une identité propre qui renvoie à une tonalité fondamentale. La compréhension réciproque des peuples suppose la compréhension de cette tonalité :

elle englobe... l'appréhension et la compréhension de leurs attitudes et tonalités d'âmes fondamentales, insondables et le plus souvent impossibles à formuler directement. Celles-ci acquièrent leur forme de référence et leur force fascinante dans la grande littérature, dans les arts plastiques et dans la pensée essentielle d'un peuple, c'est-à-dire dans la philosophie.

77. M. Heidegger, Reden und andere... (GA; 16), p. 118 (trad. fr. de N. Parfait dans Le débat: histoire - politique - société. 48, Paris, Gallimard, 1988, p. 184).

78. M. Heidegger, Introduction à la métaphysique, p. 141.

79. Martin Heidegger, M. Haar (dir.), Paris, Éditions de L'Herne (Cahiers de L'Herne; 45), 1983 , p. 71 sq. 
Il y aurait beaucoup à dire sur cette affirmation qui n'est certes pas raciste an sens de la biologie, mais qui présuppose une identité de destin, peut-être encore plus radicale, ontologique. Dans cette destinée l'individu se trouve incontestablement englobé, l'artiste ou le philosophe donnant forme à l'insondable du peuple déterminé par sa tonalité fondamentale. Chaque peuple a non seulement sa tonalité mais également, en conséquence de cette tonalité, son rang. Or, de ce point de vue, la confrontation introduit une hiérarchie: les peuples n'ont pas dans l'histoire mondiale, où l'Occident est moteur, la même importance décisive. Il n'y a pas de société des Nations mais une communauté des peuples et toute communauté suppose rang et hiérarchie. Nous voilà loin de la philosophie kantienne de l'histoire.

C'est ce que semble confirmer l'examen du rôle que Heidegger attribue au "penseur français par excellence», Descartes, qui, nous l'avons vu, invente la subjectivité, fonde les sciences modernes de la nature, inaugurant de ce fait la domination de la nature et l'instituant dans la perspective de la métaphysique. Penser cette domination de la nature exige une confrontation avec la pensée française. Mais confrontation veut dire combat, implique lutte et reconnaissance de la supériorité destinale de la pensée et du peuple allemand! Les Allemands en effet se sont élevés à la compréhension de l'Être dans son historicité, ils ont compris que l'Être ne se réduit pas à la nature considérée mathématiquement, celle des sciences modernes de la nature; ils ont compris la Geschichtlichkeit fondamentale du Dasein et sont donc plus à même que le peuple français de faire face à la menace qui pèse sur l'Occident: "peut-on alors s'étonner que depuis des années déjà, des auteurs français ${ }^{80}$ récents se sont aperçus de la nécessité de se libérer du cadre de la philosophie cartésienne» et se sont «efforcés de comprendre les penseurs allemands». Les Allemands apparaissent plus capables d'affronter la menace du déracinement total qui plane sur l'Occident tout entier! Les Allemands ont d'ailleurs reçu des Grecs une mission: comme eux ils doivent affronter l'Asiatique et s'ils veulent avoir une chance de "pénétrer dans l'espace de leur voisinage dans sa plus large étendue», ils doivent être fiers d'eux-mêmes, ce qui leur permet de savoir ce que veut dire «comprendre un autre peuple» c'est-à-dire, sans doute, de pouvoir le situer au rang qui est le sien. On éprouve un étrange sentiment en lisant la conclusion d'un tel texte, écrit pour un colloque universitaire qui se propose de situer la tâche de la philosophie allemande en rapport avec la philosophie telle qu'elle se pratique en France ${ }^{81}$.

80. On peut noter que les Allemands sont penseurs, les Français simplement «auteurs».

81. Sur les circonstances de la rédaction de cette conférence voir R. Safransky, Heidegger et son temps, Paris, Grasset, 1996, p. 457-464. 
Et ces phrases ne sont pas isolées. Dans un autre texte il affirme que ce n'est pas par hasard que les sciences modernes de la nature sont nées en France et en Angleterre (sans doute la Stimmung du commerçant affairé ou du bavard des salons). Leibniz a connu la philosophie française mais, ayant justement pu la connaître, l'a aussi surmontée et a inauguré ainsi la philosophie allemande, qui aboutit à l'idéalisme dont Heidegger comme philosophe allemand se reconnaît l'héritier: «ce n'est pas l'idéalisme allemand qui s'est effondré, c'est l'époque qui n'était plus assez forte pour demeurer à la hauteur de ce monde de l'esprit» ${ }^{82}$. Les Allemands ayant pensé l'histoire sont seuls à même de saisir l'historicité de l'Être. Ils sont le peuple des penseurs et des poètes!

On peut donc considérer que la conception que se fait Heidegger de l'Europe est germano-centriste: l'Allemagne est pensée constamment comme centre. Elle est au centre géographique de l'Europe, elle est au centre de l'histoire de l'Être, elle est au centre, entre la terre et le ciel; l'ouverture à l'être appartient à un peuple originaire qui a en charge la mémoire et le destin de l'Occident.

Heidegger substitue à l'universel de l'histoire une pensée de la singularité et de la hiérarchie; il y a une Seinsgeschichte et pas une Weltgeschichte. Notre époque a «la rage» de penser en termes d'histoire mondiale ${ }^{83}$. Or cette perspective constitue un oubli du Destin du monde, une construction artificielle objectivante. L'historicité pourrait d'ailleurs être oubliée, déniée radicalement. C'est un des risques de la guerre; il y a des peuples anhistoriques, qui peuvent gagner militairement. L'ouverture de l'Être serait alors complètement refermée par la victoire des peuples inauthentiques:

Nous savons aujourd'hui ${ }^{84}$ que le monde anglo-saxon de l'américanisme a décidé d'annihiler l'Europe, c'est-à-dire la patrie, l'origine de l'Occidental. L'originaire ne peut être liquidé. L'entrée de l'Amérique dans cette guerre planétaire n'est pas une entrée dans l'histoire, mais constitue le dernier acte américain de l'an-historicité américaine et de l'autodévastation ${ }^{85}$.

L'Occident à son extrême se retourne contre sa source, attaque l'Europe en son centre, l'Allemagne, et cherche à s'autodétruire puisque l'Amérique c'est aussi l'Occident, c'est aussi l'Européen. L'Allemagne ne peut pas perdre la guerre.

82. M. Heidegger, Introduction à la métaphysique, p. 56.

83. M. Heidegger, Qu'appelle-t-on penser?, p. 163.

84. En 1942.

85. M. Heidegger, Hölderlins Hymne «Der Ister», Francfort, Klostermann (GA; 53), 1984, p. 63 (nous traduisons). 


\section{L'absence de patrie, le dispositif, les poètes}

L'histoire concrète, effective n'obéit pas aux décisions du penseur. Cette guerre est à nouveau perdue. Mais à vrai dire, selon notre auteur, pas plus qu'en 1918, la défaite n'a rien décidé: en 1951, dans Qu'appelle-t-on penser?, Heidegger écrit: «Cette guerre mondiale n'a rien décidé si nous prenons ce mot dans le sens large et élevé qui concerne le destin de l'homme sur la terre "; «Les catégories politico-morales» de l'après-guerre sont «trop courtes ${ }^{86}$. Même après la guerre, après la défaite, l'origine demeure. L'Allemagne, plus précisément «l'Allemagne invisible» de Hölderlin repensée par Hellingrath demeure le centre secret de toute histoire; l'échec de la révolution, de la Wiederholung étant interprété à partir du développement du Gestell, dispositif dont la provenance est si profonde qu'elle domine toutes les époques et, particulièrement, la nôtre. La révolution allemande a été, elle-même, subvertie par le dispositif, elle est devenue une de ses manifestations. La terre reste donc livrée à ce processus qui déracine toute existence, détruisant la possibilité pour tout homme d'être dans une patrie; l'homme moderne est heimatlos, errant sans patrie, exilé. La terre ne peut être habitée que poétiquement. Hölderlin, le poète des Allemands, le poète de la poésie, celui dont les œuvres se trouvaient dans la poche des soldats allemands qui allaient mourir à la guerre, Hölderlin préserve le site, à partir duquel un Dieu peut venir nous sauver. La défaite ne peut décider sur ce point.

La pensée de l'Europe selon Heidegger peut-elle nous éclairer? L'Europe dans laquelle nous vivons a pris une autre voie. Est-ce celle de la dévastation de la terre et de la croissance du désert?

Robert URIAC

86. M. Heidegger, Qu'appelle-t-on penser?, p. 109. 